\title{
Habilidades sociales en los planes de formación ciudadana de escuelas de las provincias de Concepción y Arauco, región del Biobío-Chile
}

Araneda Machmar, Javiera; Montre Águila, Víctor

Habilidades sociales en los planes de formación ciudadana de escuelas de las provincias de Concepción y Arauco, región del Biobío-Chile

Revista Educación, vol. 45, núm. 2, 2021

Universidad de Costa Rica, Costa Rica

Disponible en: https://www.redalyc.org/articulo.oa?id=44066178012

DOI: https://doi.org/10.15517/revedu.v45i1.43561

\section{(c) $($ ) $\Theta(9$}

Esta obra está bajo una Licencia Creative Commons Atribución-NoComercial-SinDerivar 3.0 Internacional. 


\section{Habilidades sociales en los planes de formación ciudadana de escuelas de las provincias de Concepción y Arauco, región del Biobío-Chile}

Social Skills in Citizenship Study Plans at Schools in the Concepción and Arauco provinces region of Biobío, Chile

Javiera Araneda Machmar

Universidad Católica de la Santísima Concepción, Chile

jaranedamachmar@gmail.com

DOI: https://doi.org/10.15517/revedu.v45i1.43561

Redalyc: https://www.redalyc.org/articulo.oa? $\mathrm{id}=44066178012$

(iD) https://orcid.org/0000-0003-4770-5959

Victor Montre Águila

Universidad Católica de la Santísima Concepción, Chile

victor.montre@csc.cl

iD https://orcid.org/0000-0002-7391-1054

Recepción: 27 Agosto 2020

Aprobación: 21 Octubre 2020

\section{Resumen:}

El objetivo de la investigación fue caracterizar el desarrollo curricular de las habilidades sociales en los planes de Formación Ciudadana de escuelas de la provincia de Concepción y Arauco en la región del Biobío. La metodología del estudio fue mixta, desde la perspectiva cualitativa se utilizó un diseño hermenéutico a través de la técnica de investigación documental; cuantitativamente, el diseño es no experimental de tipo descriptivo y transversal. La muestra del estudio son los 29 planes de formación ciudadana de escuelas pertenecientes a la región del Biobío; se utilizó un muestreo por conveniencia e intencionado. Los planes fueron tratados con la técnica de análisis de contenido y descripción univariada de las variables educativas y sociodemográficas. Los resultados del estudio evidencian que la mayoría de las escuelas promueve entre 4 a 5 habilidades sociales a través de sus planes de formación ciudadana. Además, existen diferencias entre las habilidades sociales desarrolladas entre establecimientos subvencionados y municipales, provincia de Arauco y Concepción, y zona urbana y rural.

Palabras clave: Formación Ciudadana, Escuelas, Habilidades sociales.

\section{Abstract:}

This study investigates how social skills are included in citizenship studies curriculums for schools located in the Concepción and Arauco provinces pertaining to the Biobío Region in Chile. The study was conducted as a documentary based on a combined using a mixed methodology that had a qualitative perspective and hermeneutic design. Quantitatively speaking, the design was non-experimental, descriptive and cross-cutting with convenience sampling of 29 Citizenship Studies programs, specifically, in the area known as the Biobío region, The authors used a content analysis technique as well as a univariate and bivariate description of educational and socio-demographic variables. According to the study results, most of the schools promote between 4 and 5 social skills through citizenship curricular development plans. However, a marked difference was observed regarding the number of social skills developed among subsidized schools versus municipal schools, in the provinces of Arauco and Concepción, and whether or not the schools were located in urban or rural areas.

KEYWORDS: Citizenship Studies, Educational Unit, Social Skills.

\section{INTRODUCCIÓN}

La escuela corresponde a uno de los espacios de socialización más significativos para las personas, puesto que es el entorno - después del familiar- donde más se interactúa en la niñez, y es el primer espacio de socialización fuera del hogar (Pérez, 2009). En este lugar, las personas estudiantes se conocen a sí mismas, construyendo y plasmando sus identidades y prácticas ciudadanas (Muñoz y Torres, 2019). 
Los establecimientos educacionales, al otorgar este espacio de socialización, juegan un rol crucial en el proceso de enseñanza y aprendizaje de las y los estudiantes, y para hacerlo de la mejor manera, se guían con el currículum nacional. Este documento evidencia y especifica la finalidad educativa de alcanzar un desarrollo integral (Ministerio de Educación, 2019), pero a pesar de lo declarado en este, la mejora de la calidad de la educación en Chile requiere fortalecer las habilidades personales, emocionales y sociales de las y los estudiantes (Henríquez, 2021). En la misma línea, mejorar la formación ciudadana del alumnado contribuirá a la resolución de sus problemas de convivencia y, a participar activamente en la vida democrática (Mujica-Johnson, 2020). Por ello, Chile ha estado desde el año 2004 mejorando la formación cívica, siendo el último progreso la elaboración de Planes de Formación Ciudadana, gracias a la Ley 20.911 (Programa de las Naciones Unidas para el Desarrollo, 2018).

Así, el objetivo de la investigación es caracterizar el desarrollo curricular de las Habilidades Sociales [HS] en los Planes de Formación Ciudadana [PFC], puesto que entre la formación ciudadana y las habilidades sociales existen elementos que se complementan al mejorar su enseñanza, y que dentro del contexto chileno no existen estudios que describan y relacionen los datos con la evidencia latinoamericana y europea, siendo relevante evidenciar la aplicación de los planes de formación ciudadana.

El artículo está segmentado en cinco partes. Inicia dando a conocer evidencias empíricas de los últimos cinco años sobre la enseñanza de las habilidades sociales en contextos nacionales e internacionales, para luego proceder a las teorías que datan del siglo XX y que permitieron elaborar la clasificación de HS utilizada en la investigación. A continuación, se explica la metodología seleccionada: un paradigma mixto combinatorio, junto con la presentación y el tratamiento de la muestra, estudiada en la sección de los resultados, utilizando la categorización teórica previa de HS.

\section{EsTADo DE LA CUESTIÓN}

La enseñanza de habilidades sociales en la escuela favorece la resolución de problemas de convivencia y la participación democrática de los estudiantes dentro de un espacio de enseñanza y aprendizaje (Pérez, 2009; Mujica-Johnson, 2020).

A nivel europeo, se evidencia que las y los estudiantes aprenden a solucionar sus disyuntivas y a cooperar con sus compañeros a través de talleres de resolución de conflictos (Guerra, Rodríguez y Artiles, 2019), al ser acercados a realidades culturalmente distintas a las propias mediante el trabajo de competencias interculturales que potencian en ellas y ellos una visión global del mundo (Martínez-Lirola, 2018). Pero los talleres no bastan para lograr resultados a largo plazo (Kaya y Deniz 2020), pues se demostró que tras la aplicación de un programa de educación emocional no existen diferencias significativas en las y los participantes entre el inicio y el final del programa, siendo necesaria una incorporación curricular durante todo el ciclo escolar para lograr una adecuada formación de estudiantes socialmente hábiles (FonsecaPedrero, Pérez-Albéne, Ortuño-Sierra y Lucas-Molina, 2017; Carmona, Dueñas, Fernández y Salas, 2020).

En el caso de Latinoamérica, se ha comprobado una relación significativa entre el desarrollo de las Habilidades Sociales, el logro académico de las y los estudiantes, sus cualidades y sus estilos de vida (GómezAcosta, 2018; Quiñones, 2020). Al respecto, se ha comprobado que existe dependencia entre la conducta disruptiva y las habilidades sociales (Colichón, 2020). Además, el logro académico varía según la experiencia de desaprobación en el grupo-curso, las habilidades sociales y el apoyo del profesor/a percibido/a por las y los estudiantes (Fernandes, Leme, Elias y Soares, 2018; Valenzuela-Santoyo y Portillo-Peñuelas, 2018), esto indica que la presencia de factores de estrés (académicos y sociales) alteran los resultados que obtiene el alumnado, de manera que mientras más factores enfrenten las y los estudiantes, menor será su rendimiento académico, y sentirán menor satisfacción con su propia vida (Jovarini, Leme, y Correia-Zanini, 2018; El Achkar, Romera, Benevides, y Mattar, 2019; Rodríguez y Zapata, 2019). En ese sentido, quienes establecen mejores relaciones con sus compañeros/as suelen ser aquellos/as que presentan un buen desempeño (Torres, 
Fonseca y Pineda, 2017; Loria y Farfán, 2018) y para lograr establecer estas relaciones exitosas se requiere que las y los estudiantes sean socialmente hábiles (Bartholomeu y Montiel, 2017).

Desde la evidencia nacional se señala que las y los estudiantes que poseen pocas habilidades sociales tienen mayor dificultad para socializar con sus pares y profesores, y tienen probabilidades de presentar dificultades de comportamiento y experimentar fracaso académico (Milicic, Alcalay, Berger y Álamos, 2013). Sin embargo, las y los estudiantes pueden mejorar sus habilidades sociales desde la primera infancia a través del juego, y así prevenir retrasos en el desarrollo cognitivo, lingüístico, social y cultural, especialmente si su familia participa de estos juegos (Farkas, Vallotton, Strasser, Santelices y Himmel, 2017; Cáceres, Granada y Pomés, 2018).

De cierta manera, las Habilidades Sociales funcionan como un predictor del logro académico, el cual está positivamente correlacionadas con el buen desempeño, es decir, que mientras más habilidoso socialmente sea el/la estudiante, mejores serán sus resultados escolares (Repetto y Pena, 2010). También permiten predecir síntomas de salud mental, de manera más efectiva que los factores psicofísicos (Santamaría-García et al., 2020).

Además, se ha demostrado que las habilidades sociales no difieren según el género, ni la edad de las y los estudiantes (Rodríguez-Macayo, Vidal-Espinoza y Cossio-Bolaños, 2019), pero la función ejecutiva del niño/a, los entornos de socialización y la cantidad de cuidado recibido durante la infancia se relacionan positivamente con el ajuste socioemocional y la capacidad del lenguaje, generando diferencias entre las habilidades sociales de las y los jóvenes (Cuadra, Salgado, Lería y Menares, 2018; Leria-Dulčić, y SalgadoRoa, 2019; Lohndorf, Vermeer, Cárcamo, De la Harpe y Mesman, 2019).

De la evidencia recogida en las diferentes regiones, se logra reconocer que existe una escasa literatura sobre estudios que aborden currículum y habilidades sociales, concentrándose los estudios en programas de intervención, modelos de habilidades sociales y diagnósticos sobre habilidades sociales en estudiantes primarios y secundarios, pero sin ahondar en los actores que deciden el currículum de las habilidades sociales y la implementación de los planes en las escuelas.

Respecto a la cantidad de Habilidades Sociales, cada uno de los estudios previamente mencionados utiliza su propia clasificación y listado de habilidades, sin embargo, ninguno se concentra en alguna región del país en específico ni las abarca dentro de la enseñanza de la ciudadanía en los establecimientos escolares.

En conclusión, tanto las investigaciones europeas como las latinoamericanas coinciden en que las habilidades sociales están vinculadas con el rendimiento académico de las y los estudiantes, siendo necesario incorporarlas a lo largo de todo el ciclo escolar, considerando los entornos de socialización y aprendizaje, pero existe una vacío de investigación sobre la conexión entre las habilidades sociales y el currículum escolar (planes de formación ciudadana).

\section{Marco teórico}

A nivel mundial, el estudio y origen de las Habilidades Sociales nace en la segunda mitad del siglo XX, específicamente alrededor de 1970 (Goldstein, Sprafkin, Gershaw, y Klein, 1989). De forma posterior, el concepto se desvincula de lo ejecutivo y se relaciona a la Psicología Social, la cual corresponde a una rama de la psicología que se dedica al análisis de las interacciones sociales. Maisto y Morris (2009) la definen como un estudio científico que se encarga de examinar la influencia que reciben los pensamientos, sentimientos y conductas de una persona, por parte de la conducta, -ya sea esta real, imaginaria o inferida- de otra persona.

En el ámbito educacional, la teoría Bioecológica de Urie Bronfenbrenner (1979) complementa el estudio psicosocial, pues incluye la idea de un contexto en donde las personas son influenciadas por elementos o situaciones presentes en sus sistemas sociales y culturales (Woolfolk, 2010).

En el contexto nacional, el estudio de las Habilidades Sociales está precedido por el de las competencias blandas, que comenzaron a introducirse en el currículum nacional a partir de 1990, con la incorporación de las competencias que serían necesarias para el siglo XXI (Bellei y Morawietz, 2016). 
Las habilidades sociales pueden ser definidas desde la psicología social (Maisto y Morris, 2009) como una capacidad que tienen las personas sin necesidad de recurrir a un entrenamiento, es decir, que las habilidades se obtendrían de forma natural e innata. Desde el ámbito conductual, Caballo e Irurtia (2011) explican que el concepto de habilidad hace referencia a las conductas interpersonales, correspondientes a un conjunto de capacidades de actuación aprendidas. Para Costales, Fernández y Macías (2014), el concepto de Habilidades Sociales integra características de la psicología personal y la conductual. Suponen que la conducta interpersonal corresponde a un conjunto de capacidades de actuaciones aprendidas que se ven determinadas por factores ambientales, personales, y de la interacción de ambos factores.

TABLA 1

Relación entre la clasificación y los tipos de habilidades sociales

\begin{tabular}{|c|c|c|c|c|c|}
\hline & CLASIFICACIÓN & & & & \\
\hline HABILIDAD SOCIAL & $\begin{array}{l}\text { Habilidades } \\
\text { Sociales Escolares }\end{array}$ & $\begin{array}{l}\text { Goldstein, Sprafkin, } \\
\text { Gershaw y Klein }\end{array}$ & Caballo e Irurtia & UNESCO & Big Five \\
\hline Empatia & $\mathrm{X}$ & $\mathrm{X}$ & $\mathrm{X}$ & $\mathrm{X}$ & $\mathrm{x}$ \\
\hline Autocontrol & $\mathrm{x}$ & $\mathrm{X}$ & & & $\mathrm{x}$ \\
\hline Cortesía & $\mathrm{x}$ & $\mathrm{X}$ & & & $\mathrm{x}$ \\
\hline Asertividad & $\mathrm{X}$ & $\mathrm{X}$ & $\mathrm{X}$ & & $\mathrm{x}$ \\
\hline Acercamiento Afectivo & $\mathrm{X}$ & $\mathrm{X}$ & $\mathrm{X}$ & $\mathrm{x}$ & $\mathrm{x}$ \\
\hline Desarrollo Social & $\mathrm{X}$ & $\mathrm{X}$ & $\mathrm{X}$ & $\mathrm{X}$ & $\mathrm{X}$ \\
\hline
\end{tabular}

Fuente: Elaboración a partir de Gonçalves, Romera y Vieira (2018), Goldstein et al. (1989), Caballo e Irurtia (2011), Scott (2015) y OECD (2018).

Otra definición del concepto es aquella proveniente del enfoque de aprendizaje social, que considera que las Habilidades Sociales son conductas asertivas aprendidas a través de la observación de modelos (Segura, 2002). Desde la perspectiva de la Teoría Bioecológica, las Habilidades Sociales se refieren al conjunto diverso de comportamientos del repertorio de una persona que pueden contribuir a su competencia social, conduciendo a relaciones saludables y productivas (Gonçalves et al., 2018).

En conclusión, una Habilidad Social se comprenderá como el conjunto de conductas asertivas necesarias para interactuar, que surgen de manera adecuada a cada situación, condicionadas por el contexto tanto externo como interno de la persona. Asimismo, una conducta será socialmente habilidosa si es acorde al nivel de madurez moral y cognitivo del individuo, y le facilita el establecimiento de relaciones interpersonales saludables y productivas. Reconociendo la existencia de 6 habilidades sociales vinculadas a la empatía, autocontrol, cortesía, asertividad, acercamiento afectivo y el desarrollo social (ver Tabla 1) y que tienen relación con las clasificaciones propuestas por diferentes autores/as (Gonçalves et al.,2018; Goldstein et al., 1989; Caballo y Irurtia., 2011; OECD, 2018; Scott, 2015).

\section{Procedimientos metodológicos}

El estudio presenta un paradigma mixto combinatorio (Bericat, 1998), desarrollando un enfoque cuantitativo a través de un diseño no experimental, transversal y descriptivo (Hernández-Sampieri y Mendoza, 2018), por otra parte, la sección cualitativa presenta un enfoque interpretativo a través de una tradición hermenéutica con un método de investigación documental (Flick, 2004; Vasilachis, 2006).

La muestra de la investigación consiste en 29 escuelas de la región del Biobío, utilizando como unidad de análisis los Planes de Formación Ciudadana de los establecimientos que los elaboraron, caracterizándose por ser una muestra no representativa e intencionada (ver Tabla 2). Los criterios de inclusión de la muestra fueron escuelas de la provincia de Concepción y Arauco de la región del Biobío, pertenecientes a un área rural o urbana y que impartieran educación primaria-secundaria. 
La recolección de los planes de Formación Ciudadana está enmarcada en el proyecto de Colaboración de la Universidad de Concepción, con establecimientos educativos de la provincia de Concepción y Arauco en temáticas de Ciudadanía, Democracia y Derechos Humanos.

TABLA 2

Caracterización de la muestra de estudio

$\begin{array}{llll}N^{\circ} & \text { Establecimiento } & \text { Comuna } & \text { Dependencia } \\ 1 & \text { A-Lafken } & \text { Penco } & \text { P. Subvencionado } \\ 2 & \text { Alberto Blest Gana } & \text { San Pedro de la Paz P. Subvencionado } \\ 3 & \text { Antonio Salamanca Morales } & \text { Coronel } & \text { P. Subvencionado } \\ 4 & \text { Arnoldo Ebensperger } & \text { Lebu } & \text { Municipal } \\ 5 & \text { Arturo Ebensperger } & \text { Lebu } & \text { Municipal } \\ 6 & \text { Blanca Estela } & \text { Concepción } & \text { Municipal } \\ 7 & \text { Cacique Francisco Melin } & \text { Cañete } & \text { Municipal } \\ 8 & \text { California } & \text { Tomé } & \text { Municipal } \\ 9 & \text { CEIA } & \text { Coronel } & \text { Municipal } \\ 10 & \text { Claudio Flores Soto } & \text { Los Álamos } & \text { Municipal } \\ 11 & \text { Colico Sur } & \text { Curanilahue } & \text { Municipal } \\ 12 & \text { Galvarino } & \text { San Pedro de la Paz Municipal } \\ 13 & \text { José Manuel Balmaceda } & \text { Concepción } & \text { Municipal } \\ 14 & \text { La Esperanza } & \text { Chiguayante } & \text { Municipal } \\ 15 & \text { La Granja } & \text { Cañete } & \text { Municipal } \\ 16 & \text { Las Américas } & \text { Talcahuano } & \text { Municipal } \\ 17 & \text { Leoncio Araneda Figueroa } & \text { Cañete } & \text { Municipal } \\ 18 & \text { Monseïor Alfredo Silva Santiago } & \text { Concepción } & \text { P. Subvencionado } \\ 19 & \text { Nahuelquin } & \text { Cabrero } & \text { Municipal } \\ 20 & \text { Padre Manuel D'alzon } & \text { Lota } & \text { P. Subvencionado } \\ 21 & \text { Pehuén } & \text { Lebu } & \text { Municipal } \\ 22 & \text { Pencopolitano } & \text { Penco } & \text { Municipal } \\ 23 & \text { Porvenir } & \text { Chiguayante } & \text { P. Subvencionado } \\ 24 & \text { San Felipe } & \text { Arauco } & \text { Municipal } \\ 25 & \text { San Francisco } & \text { Talcahuano } & \text { Municipal } \\ 26 & \text { San Juan Bautista } & \text { Hualqui } & \text { Municipal } \\ 27 & \text { San Luis de Potosi } & \text { Lota } & \text { Municipal } \\ 28 & \text { San Marcos } & \text { Coronel } & \text { P. Subvencionado } \\ 29 & \text { Los Álamos } & \text { Municipal } \\ & & \end{array}$

Fuente: Elaboración propia

El tratamiento de la información fue construida a través de la técnica de análisis de contenido en los Planes de Formación Ciudadana, utilizando una categorización teórica previa de 6 habilidades sociales fundamentada en la propuesta de Gonçalves, et al., (2018). Luego, al identificar las habilidades sociales presentes en los planes de Formación Ciudadana de cada establecimiento, se construyó una tabla de variables educativas (Empatía, Autocontrol, Asertividad, Cortesía, Acercamiento y Desarrollo social) y sociodemográficas (tipo de dependencia, tipo de plan, localización y provincia) en el programa estadístico SPSS 19 y se procedió a realizar un análisis estadístico univariado (frecuencia, moda y porcentaje) siendo visualizado a través de gráficos de sectores y barra.

\section{RESULTADOS Y DISCUSIÓN}

La Tabla 3 presenta en la primera columna, de izquierda a derecha, la asociación de cada PFC a un número que lo identifica dentro de las 29 escuelas de la región del Biobío. Las demás columnas representan a cada 
Habilidad Social Escolar y las celdas representan la intersección de los PFC con las HSE. Las equis en las celdas indican la presencia de la HSE en el PFC, por lo tanto, aquellas celdas sin equis indican que la HSE no está presente en el PFC.

TABLA 3

Presencia de Habilidades Sociales en los Planes de Formación Ciudadana de Establecimientos Educativos

\begin{tabular}{|c|c|c|c|c|c|c|}
\hline $\begin{array}{l}\mathrm{N}^{\circ} \mathrm{PF} \\
\mathrm{C}\end{array}$ & Empatía & Autocontrol & Cortesia & Asertividad & $\begin{array}{l}\text { Acercamiento } \\
\text { Afectivo }\end{array}$ & $\begin{array}{l}\text { Desarrollo } \\
\text { Social }\end{array}$ \\
\hline 1 & $\mathrm{x}$ & & $\mathrm{X}$ & $\mathrm{X}$ & $\mathrm{x}$ & $\mathrm{X}$ \\
\hline 2 & $\mathrm{x}$ & $\mathrm{X}$ & $\mathrm{X}$ & $\mathrm{X}$ & $\mathrm{X}$ & $\mathrm{X}$ \\
\hline 3 & $\mathrm{x}$ & & $\mathrm{X}$ & $\mathrm{X}$ & & $\mathrm{x}$ \\
\hline 4 & $\mathrm{x}$ & & $\mathrm{X}$ & $\mathrm{X}$ & & $\mathrm{X}$ \\
\hline 5 & $\mathrm{x}$ & $\mathrm{X}$ & $\mathrm{X}$ & $\mathrm{X}$ & $\mathrm{X}$ & $\mathrm{X}$ \\
\hline 6 & $\mathrm{x}$ & $\mathrm{x}$ & $\mathrm{X}$ & $\mathrm{X}$ & $\mathrm{x}$ & $\mathrm{X}$ \\
\hline 7 & $\mathrm{X}$ & & $\mathrm{X}$ & & $\mathrm{X}$ & \\
\hline 8 & $\mathrm{X}$ & $\mathrm{X}$ & $\mathrm{X}$ & $\mathrm{X}$ & & $\mathrm{X}$ \\
\hline 9 & & & & $\mathrm{X}$ & & $\mathrm{X}$ \\
\hline 10 & $\mathrm{x}$ & $\mathrm{X}$ & $\mathrm{X}$ & $\mathrm{X}$ & & $\mathrm{X}$ \\
\hline 11 & $\mathrm{X}$ & & & & & $\mathrm{X}$ \\
\hline 12 & $\mathrm{X}$ & & $\mathrm{X}$ & $\mathrm{X}$ & & $\mathrm{X}$ \\
\hline 13 & $\mathrm{x}$ & $\mathrm{X}$ & $\mathrm{X}$ & $\mathrm{X}$ & $\mathrm{X}$ & $\mathrm{X}$ \\
\hline 14 & $\mathrm{x}$ & $\mathrm{X}$ & $\mathrm{X}$ & $\mathrm{X}$ & & $\mathrm{X}$ \\
\hline 15 & $\mathrm{x}$ & $\mathrm{X}$ & & $\mathrm{X}$ & & $\mathrm{X}$ \\
\hline 16 & $\mathrm{x}$ & $\mathrm{X}$ & $\mathrm{X}$ & $\mathrm{X}$ & & $\mathrm{X}$ \\
\hline 17 & $\mathrm{x}$ & $\mathrm{X}$ & $\mathrm{X}$ & $\mathrm{X}$ & & $\mathrm{X}$ \\
\hline 18 & $\mathrm{x}$ & $\mathrm{x}$ & $\mathrm{X}$ & $\mathrm{X}$ & $\mathrm{X}$ & $\mathrm{X}$ \\
\hline 19 & $\mathrm{X}$ & $\mathrm{X}$ & $\mathrm{X}$ & $\mathrm{X}$ & $\mathrm{X}$ & $\mathrm{X}$ \\
\hline 20 & $\mathrm{x}$ & $\mathrm{X}$ & $\mathrm{X}$ & $\mathrm{X}$ & & \\
\hline 21 & $\mathrm{x}$ & $\mathrm{x}$ & $\mathrm{X}$ & & & $\mathrm{x}$ \\
\hline 22 & $\mathrm{X}$ & $\mathrm{X}$ & $\mathrm{X}$ & & $\mathrm{X}$ & $\mathrm{X}$ \\
\hline 23 & $\mathrm{x}$ & $\mathrm{x}$ & $\mathrm{X}$ & & $\mathrm{X}$ & $\mathrm{X}$ \\
\hline 24 & $\mathrm{X}$ & & $\mathrm{X}$ & $\mathrm{X}$ & & $\mathrm{X}$ \\
\hline 25 & $\mathrm{X}$ & $\mathrm{X}$ & $\mathrm{X}$ & $\mathrm{X}$ & & $\mathrm{X}$ \\
\hline 26 & $\mathrm{x}$ & & $\mathrm{X}$ & $\mathrm{X}$ & & $\mathrm{X}$ \\
\hline 27 & $\mathrm{x}$ & & $\mathrm{X}$ & $\mathrm{X}$ & & $\mathrm{X}$ \\
\hline 28 & & & & & & $\mathrm{X}$ \\
\hline 29 & $\mathrm{X}$ & & $\mathrm{X}$ & $\mathrm{X}$ & $\mathrm{X}$ & $\mathrm{X}$ \\
\hline
\end{tabular}

Fuente: Elaboración Propia

En la Tabla 4 se presenta la cantidad de Habilidades Sociales Escolares que desarrolla cada establecimiento en su Formación Ciudadana. Se observa que solo uno (correspondiente al 3\% de los establecimientos) impulsa una única Habilidad Social Escolar en su proyecto, dos establecimientos (7\%) consideran 2 HSE, uno (3\%) trabaja 3 HSE, ocho escuelas (28\%) incorporan $4 \mathrm{HSE}$, once (38\%) promueven 5 HSE, y seis (21\%) elaboraron su PFC considerando el total de las Habilidades Sociales Escolares. 
TABLA 4

Cantidad de Habilidades Sociales desarrolladas por Planes de Formación Ciudadana en Establecimientos Educativos

Leyenda

\begin{tabular}{cllll}
\multicolumn{5}{c}{ Frecuencia Porcentaje Porcentaje válido Porcentaje acumulado } \\
Una & 1 & 3,4 & 3,4 & 3,4 \\
Dos & 2 & 6,9 & 6,9 & 10,3 \\
Tres & 1 & 3,4 & 3,4 & 13,8 \\
Habilidad Social Cuatro 8 & 27,6 & 27,6 & 41,4 \\
Cinco & 11 & 37,9 & 37,9 & 79,3 \\
Seis & 6 & 20,7 & 20,7 & 100,0 \\
Total & 29 & 100,0 & 100,0 &
\end{tabular}

Fuente: Elaboración propia

Además, la Tabla 5 presenta la asociación entre el Total de Habilidades Sociales Escolares con la localización del establecimiento al cual pertenece el PFC. Se observa que, de los PFC pertenecientes a una localización urbana, en relación con el total de establecimientos: el 3\% impulsa $1 \mathrm{HSE}$, el 7\% 2 HSE, el 24\% 4 HSE, un 35\% 5 HSE y un 21\% 6 HSE. Sobre los PFC de establecimientos rurales: 3\% promueve 3 HSE, $3 \% 4$ HSE y $3 \% 5$ HSE.

De las unidades estudiadas, gran parte de los planes de formación ciudadana promueven 4 o 5 HS y un rango menor entre 1 a 3 HS, siendo influenciado por los objetivos de la ley 20.911, que solo hacen referencia a cuatro HSE: Asertividad, Empatía, Desarrollo social y Cortesía, sin presentar exigencias sobre el Autocontrol ni el Acercamiento afectivo (Ministerio de Educación, 2016).

Ante esto, las escuelas, como espacios de socialización, tienen un rol relevante en el proceso de desarrollo de habilidades sociales (Ministerio de Educación, 2019), siendo necesario fomentar que todas las escuelas promuevan las 6 HS. Posibilitando mejoras en la calidad educativa, fortaleciendo las habilidades personales, emocionales y sociales de los educandos (Henríquez, 2021; Mujica-Johnson, 2020).

Ante la existencia de escuelas que tienen una escasa promoción de HS, es posible señalar que las y los estudiantes que tienen pocas habilidades sociales presentan dificultades de comportamiento y experimentan fracaso académico (Milicic et al, 2013; Repetto y Pena, 2010; Santamaría-García et al., 2020). 
TABLA 5

Asociación entre las HSE y la Localización de los Establecimientos

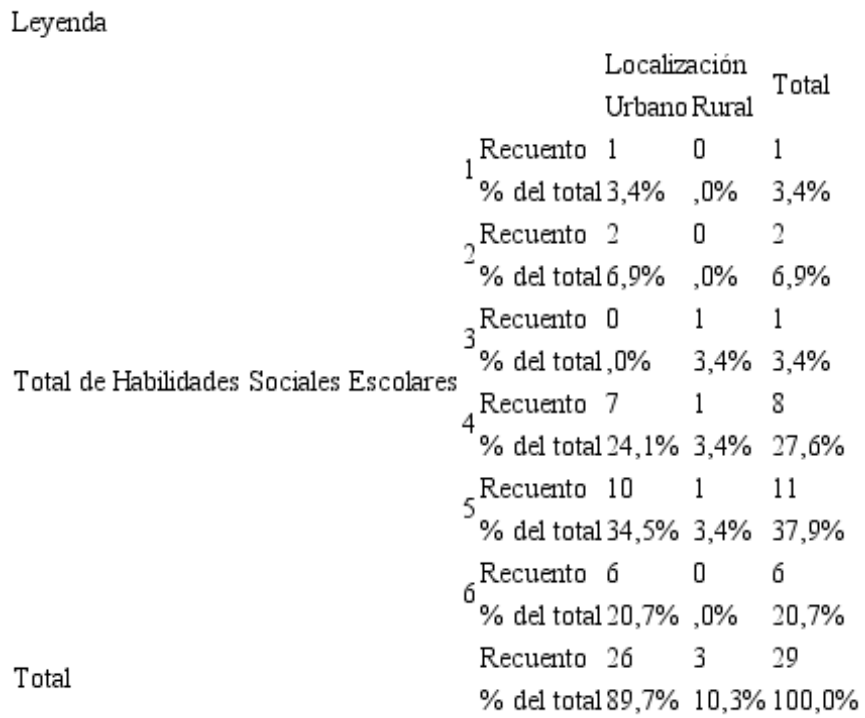

TABLA 6

Asociación entre las HSE y el Tipo de Plan de Formación Ciudadana Leyenda

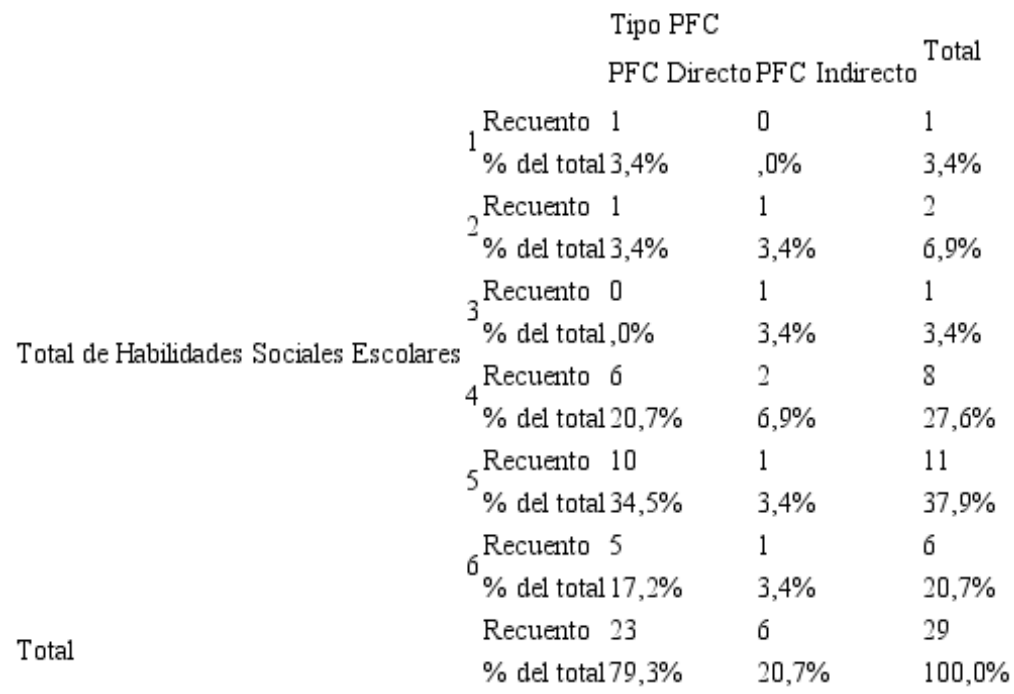

Se evidencia en la Tabla 6 que es más frecuente que aquellos establecimientos que elaboran PFC directo desarrollen 5 HSE y aquellos que incorporan sus PFC en sus PEI trabajen 4 Habilidades Sociales Escolares. 
En la Tabla 7 se presenta la asociación entre la provincia a la cual pertenece el establecimiento que elaboró el PFC y la presencia del Total de Habilidades Sociales Escolares en el documento. Se observa en las unidades de la provincia de Concepción, un desarrollo de: el 3\% de una HSE, el 3\% de dos HSE, el 14\% de cuatro HSE, el $28 \%$ de cinco HSE y el 17\% de seis HSE. De los establecimientos que pertenecen a la provincia de Arauco: el 3\% desarrolla dos HSE, el 3\% tres HSE, el 14\% cuatro HSE, el 10\% cinco HSE y el 3\% seis HSE.

También se demuestra que los establecimientos Municipales promueven 5 HSE en sus PFC, mientras que los Particulares Subvencionados están entre 4 y 6 HSE (ver Tabla 8).

TABLA 7

Asociación entre las HSE y la Provincia del Establecimiento

\begin{tabular}{|c|c|c|c|c|}
\hline \multicolumn{5}{|l|}{ Leyenda } \\
\hline & & \multicolumn{2}{|c|}{ Provincia } & \\
\hline & & \multicolumn{3}{|c|}{ Concepción Arauco ${ }^{1 \text { otal }}$} \\
\hline \multirow{12}{*}{ Total de Habilidades Sociales Escolares } & Recuento & 1 & 0 & 1 \\
\hline & $\%$ del tota & $13,4 \%$ &, $0 \%$ & $3,4 \%$ \\
\hline & Recuento & 1 & 1 & 2 \\
\hline & \% del tota & $13,4 \%$ & $3,4 \%$ & $6,9 \%$ \\
\hline & ${ }_{3}$ Recuento & 0 & 1 & 1 \\
\hline & $\%$ del tota & $1,0 \%$ & $3,4 \%$ & $3,4 \%$ \\
\hline & ${ }_{4}$ Recuento & 4 & & 8 \\
\hline & ${ }^{4} \%$ del tota & $113,8 \%$ & $13,8 \%$ & $27,6 \%$ \\
\hline & ${ }_{5}$ Recuento & 8 & 3 & 11 \\
\hline & \% del tota & $127,6 \%$ & $10,3 \%$ & $37,9 \%$ \\
\hline & $\sigma_{6}^{\text {Recuento }}$ & 5 & 1 & 6 \\
\hline & $\%$ del tota & $117,2 \%$ & $3,4 \%$ & $20,7 \%$ \\
\hline \multirow{2}{*}{ Total } & Recuento & 19 & 10 & 29 \\
\hline & $\%$ del tota & $165,5 \%$ & $34,5 \%$ & $100,0 \%$ \\
\hline
\end{tabular}

Fuente: Elaboración propia

Independiente de la localización zonal (urbano o rural) o dependencia de los establecimientos, es importante recordar que todos los establecimientos educativos se rigen por las mismas bases curriculares. Esto implica fomentar las habilidades sociales para establecer mejores relaciones con sus compañeros/as (Torres, Fonseca y Pineda, 2017; Loria y Farfán, 2018; Bartholomeu y Montiel, 2017).

Además, la aplicación de propuestas curriculares lograría formar adecuadamente las habilidades sociales de las y los estudiantes (Fonseca-Pedrero, Pérez-Albéne, Ortuño-Sierra y Lucas-Molina, 2017; Carmona, Dueñas, Fernández y Salas, 2020). En cambio, si no es aplicado con una lógica transversal, las intervenciones seccionadas no tienen efecto sobre el desarrollo emocional de las personas (Kaya y Deniz 2020). 
TABLA 8

Asociación entre las HSE y la Dependencia del Establecimiento

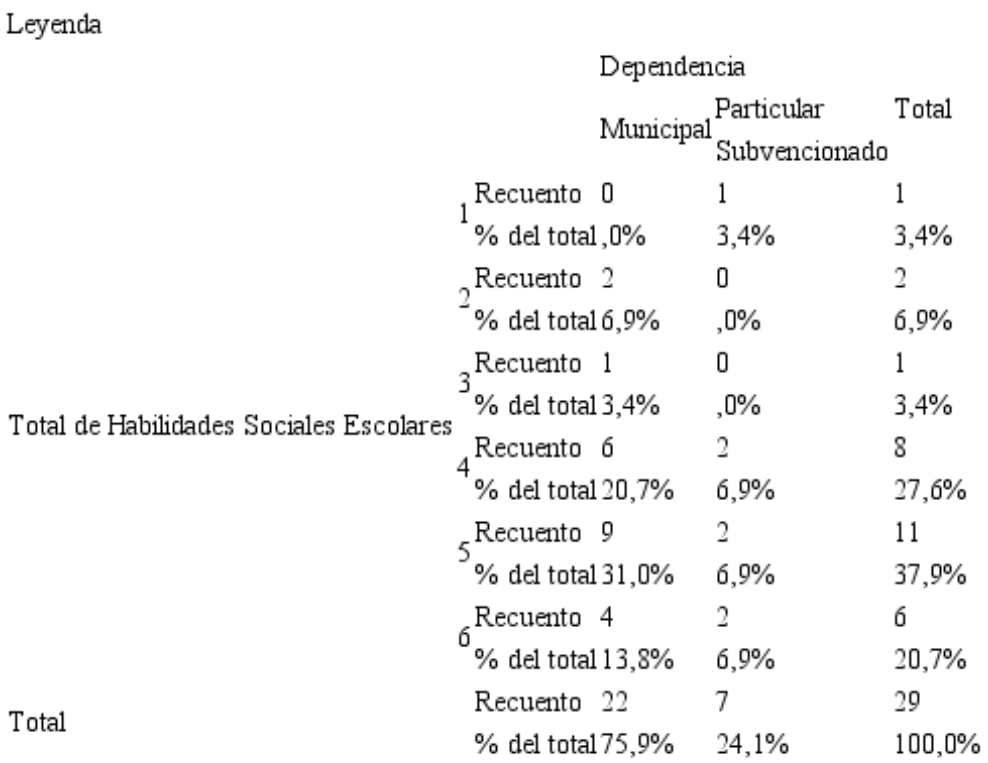

Fuente: Elaboración propia

\section{Conclusiones}

Un camino para modificar la realidad evidenciada es la promoción de talleres sobre resolución de conflictos y que permitan una visión cultural del mundo (Guerra, Rodríguez y Artiles, 2019; Martínez-Lirola, 2018), debiendo ser incorporados a los planes curriculares (Fonseca-Pedrero et al., 2017).

En consecuencia, si este nuevo documento es aplicado en las escuelas, ¿cómo se espera que las y los docentes sepan llevarlo adecuadamente a la sala de clases, si este no presenta un vínculo concreto con los otros documentos? o ¿cómo saben las y los docentes que sus estudiantes han desarrollado el objetivo acorde a lo esperado, si no tienen algún referente, pauta o punto de comparación, más que saber reconocerlo desde su propia percepción y experiencia?

Ante esas situaciones, las y los profesores tienden a basarse en sus propias experiencias para explicar algo que para ellos/as tiene sentido, pero que termina distanciándose y descontextualizándose aún más para las y los estudiantes (Muñoz y Torres, 2014).

Teóricamente, los planes presentan sus objetivos y las escuelas proponen actividades para lograrlos, pero en estos planes no se observa alguna medición, regulación o punto de comparación que permita comprobar en la práctica que estas metas se están logrando. Además, es necesario profundizar en las decisiones de quienes construyen los planes de formación ciudadana al elaborar las bases curriculares.

La consideración de HS en los objetivos en los planes puede deberse a que es un instrumento relativamente nuevo para el Sistema Educativo Chileno, en el sentido de que los planes ingresaron al sistema el año 2016, y los resultados de PISA del año 2018, no presentaron los impactos esperados del plan, ya que el informe aún 
concluye que es necesario mejorar y aumentar las habilidades interpersonales e intrapersonales de las y los estudiantes (OECD, 2018).

La investigación presenta limitaciones debido a su naturaleza metodológica de presentar una fotografía de los planes de formación ciudadana. Además, no vincula el componente curricular con los componentes evaluativos y didácticos de las habilidades sociales. Esto presenta una oportunidad de investigación a futuro para aumentar la evidencia sobre el desarrollo de habilidades sociales relacionados a la ley 20.911.

Finalmente, se espera que progresivamente las mejoras en los planes conlleven a una formación ciudadana transversal, integrada y que presente un mayor desarrollo de HS en la escuela. Esto debería ser observable en los próximos resultados de PISA, ya que las próximas generaciones medidas estarían logrando el desafío de desarrollarse intra e interpersonalmente.

\section{REFERENCIAS}

Bartholomeu, D., y Montiel, J. (2017). Behavioral and personality predictors of acceptance and rejection in University. Paidéia (Ribeirão Preto), 27(68), 272-280. doi: https://dx.doi.org/10.1590/1982-43272768201704

Bellei, C., y Morawietz, L. (2016). Contenido fuerte, herramientas débiles: Competencias del siglo XXI en la reforma educativa chilena. En H. G. Education, C. K. Chung, y F. M. Reimers (Eds.), Teaching and Learning for the Twenty-First Century Educational Goals, Policies, and Curricula from Six Nations (pp 121-162). Cambridge, Massachusetts, Estados Unidos: Harvard Education Publishing Group.

Bericat, E. (1998). La integración de los métodos cuantitativo y cualitativo en la investigación social. Barcelona: Ariel Sociología.

Bronfenbrenner, U. (1979). La ecología del desarrollo humano. Buenos Aires: Ediciones Paidós.

Caballo, V. E., y Irurtia, M. J. (2011). Entrenamiento en Habilidades Sociales. En F. J. Labrador Encinas, Técnicas de Modificación de Conducta (pp. 573-592). España: Ediciones Pirámide.

Cáceres, F., Granada, M., y Pomés, M. (2018). Inclusión y Juego en la Infancia Temprana. Revista latinoamericana de educación inclusiva, 12(1), 181-198. doi: https://doi.org/10.4067/s0718-73782018000100181

Carmona, S., Dueñas, C., Fernández, P., y Salas, B. (2020). Competencias emocionales de las futuras personas docentes: un estudio sobre los niveles de inteligencia emocional y empatía. Revista Educación, 44(2), 76-90. doi: https:/ /doi.org/10.15517/revedu.v44i2.38438

Colichón Chiscul, M. E. (2020). Inteligencia emocional y habilidades sociales en la conducta disruptiva de estudiantes del nivel secundario | Emotional intelligence and social skills in the disruptive behavior of secondary school students. ESPIRAL. CUADERNOS DEL PROFESORADO, 13(26), 29-40. doi: https://doi.org/10.25115/e cp.v13i26.2679

Costales, Y., Fernández, A., y Macías, C. (2014). Algunas consideraciones teóricas sobre las habilidades sociales. Revista Información Cientifica, 87(5), 949-959. Recuperado de https://www.redalyc.org/articulo.oa?id=5517/551757 255019

Cuadra, D., Salgado, J., Lería, F., y Menares, N. (2018). Teorías subjetivas en docentes sobre el aprendizaje y desarrollo socioemocional: Un estudio de caso. Revista Educación, 42(2), 250-271. doi: https://doi.org/10.15517/revedu .$v 42 \mathrm{i} 2.25659$

El Achkar, A., Romera, V., Benevides, A., y Mattar, M. (2019). Life Satisfaction and Academic Performance of Elementary School Students. Psico-USF, 24(2), 323-335. doi: https://doi.org/10.1590/1413-82712019240209

Farkas, C., Vallotton, C. D., Strasser, K., Santelices, M. P., y Himmel, E. (2017). Socioemotional skills between 12 and 30 months of age on Chilean children: When do the competences of adults matter? Infant Behavior and Development, 49, 192-203.doi: https://doi.org/10.1016/j.infbeh.2017.09.010

Goldstein, A., Sprafkin, R., Gershaw, J., y Klein, P. (1989). Habilidades sociales y autocontrol en la adolescencia. (A. Sàenz, Trad.) Barcelona, España: Ediciones Martínez Roca 
Gómez-Acosta, C. (2018). Factores psicológicos predictores de estilos de vida saludable. Revista de Salud Pública, 20, 155-162. doi: https://doi.org/10.15446/rsap.V20n2.50676

Gonçalves, M., Romera, V., y Vieira, N. (2018). Influence of Social Skills and Stressors on Academic Achievement in the Sixth-Grade. Paidéia (Ribeirão Preto), 28, e2819. doi: https://doi.org/10.1590/1982-4327e2819

Guerra, M., Rodríguez, J., y Artiles, J. (2019). Aprendizaje colaborativo: experiencia innovadora en el alumnado universitario. Revista de estudios y experiencias en educación, 18(36), 269-281. doi: https://dx.doi.org/10.21703 /rexe.20191836guerra5

Fernandes, L., Leme, V., Elias, L., y Soares, A. (2018). Preditores do Desempenho Escolar ao final do Ensino Fundamental: Histórico de Reprovação, Habilidades Sociais e Apoio Social. Trends in Psychology, 26(1), 215-228. doi: https://dx.doi.org/10.9788/tp2018.1-09pt

Flick, U. (2004). Introducción a la investigación cualitativa. Madrid: Ediciones Morata.

Fonseca-Pedrero, E., Pérez-Albéne, A., Ortuño-Sierra, J., y Lucas-Molina, B. (2017). Efectos de una intervención de educación emocional en alumnos de un programa universitario para mayores. Universitas Psychologica, 16(1), 286-296. doi: https://dx.doi.org/10.11144/Javeriana.upsy16-1.eiee

Henríquez, C. (marzo de 2021). Las habilidades socioemocionales en la escuela. Revista Educar. Recuperado de https ://www.grupoeducar.cl/revista/edicion-219/las-habilidades-socioemocionales-en-la-escuela/

Hernández-Sampieri, R., y Mendoza, C. (2018). La Metodología de la Investigación. Las Rutas Cuantitativa, Cualitativa y Mixta. Ciudad de México: Mc Graw Hill Education.

Jovarini, N., Leme, V., y Correia-Zanini, M. (2018). Influence of Social Skills and Stressors on Academic Achievement in the Sixth-Grade. Paidéia (Ribeirão Preto), 28, e2819. doi: https://dx.doi.org/10.1590/1982-4327e2819

Kaya, I., y Deniz, M. E. (2020). The effects of life skills education program on problem behaviors and social skills of 4-year-old preschoolers. Elementary Education Online, 19(2), 612-623. doi: https://doi.org/10.17051/ilkonlin e.2020.692983

Leria-Dulčić, F., y Salgado-Roa, A. (2019). Efecto del clima social escolar en la satisfacción con la vida en estudiantes de primaria y secundaria. Revista Educación, 43(1), 364-379. doi: https://doi.org/10.15517/REVEDU.V43I1 .30019

Lohndorf, R., Vermeer, J., Cárcamo, A., De la Harpe, C., y Mesman, J. (2019). Preschoolers' problem behavior, prosocial behavior, and language ability in a Latin-American context: The roles of child executive functions and socialization environments. Early Childhood Research Quarterly, 48, 36-49. doi: https://doi-org.ezpbibliotecas .udec.cl/10.1016/j.ecresq.2019.02.005

Loria, M., y Farfán, M. (2018). Estudio exploratorio de las competencias sociales en niños de una escuela primaria de la Ciudad de Mérida/Exploratory study of social skills in children from an elementary school in the city of Merida, México. Magister, 29(2), 1-8. doi: https://doi.org/10.17811/msg.29.2.2017

Martínez-Lirola, M. (2018). La importancia de introducir la competencia intercultural en la educación superior: Propuesta de actividades prácticas. Revista Electrónica Educare, 22(1), 40-58. doi: https://dx.doi.org/10.15359 /ree.22-1.3

Maisto, A. A., y Morris, C. G. (2009). Psicología. (L. Gaona Figueroa, Ed.) México: Pearson Educación.

Milicic, N., Alcalay, L., Berger, C., y Álamos, P. (2013). Aprendizaje socioemocional en estudiantes de quinto y sexto grado: presentación y evaluación de impacto del programa BASE. Ensaio: Avaliação e Políticas Públicas em Educação, 21(81), 645-666. Recuperado de https://www.redalyc.org/articulo.oa?id=3995/399538147002

Ministerio de Educación. (28 de Marzo de 2016). Ley N²0.911. Santiago, Chile.

Ministerio de Educación. (27 de Junio de 2019). Ley No20.370. Recuperado de https://www.bcn.cl/leychile/navega r?idNorma $=1006043$

Mujica-Johnson, N. (2020). Análisis crítico del currículo escolar en Chile en función de la justicia social. Revista Electrónica Educare, 24(1), 1-14. doi: http://doi.org/10.15359/ree.24-1.25

Muñoz, C., y Torres, B. (2014). La formación ciudadana en la escuela: Problemas y desafíos. Revista Electrónica Educare, 18(2), 233-245. doi: http://dx.doi.org/10.15359/ree.18-2.12 
Javiera Araneda Machmar, et al. Habilidades sociales en los planes de formación ciudadana de escu...

Muñoz, C., y Torres, B. (2019). Escuela y formación ciudadana: Temas, escenarios y propuestas para su desarrollo (Primera ed.). Concepción, Chile: Universidad de Concepción.

OECD (2018). Social and emotional skills for student success and well-being. Education Working Papers, 173, 1-136. doi: https://doi.org/10.1787/db1d8e59-en

Programa de las Naciones Unidas para el Desarrollo. (2018). Sobre el Plan de Formación Ciudadana. Ciudadanía y Escuela. Recuperado de https://www.ciudadaniayescuela.cl/sobre-el-plan/

Quiñones, T. (2020). Habilidades sociales y estrategias didácticas para la formación del liderazgo desde la educación básica. Revista Educación, 44(2), 651-665. doi: https://doi.org/10.15517/revedu.v44i2.40270

Repetto, E., y Pena, M. (2010). Las competencias socioemocionales como factor de calidad en la educación. Revista Iberoamericana sobre Calidad, Eficacia y Cambio en Educación (RINACE), 8(5), 82-95. Recuperado de https:/ /www.redalyc.org/pdf/551/55119084006.pdf

Rodríguez, A., y Zapata, E. (2019). Formación para la tolerancia, autorregulación de las emociones. Revista Educación, 43(2), 433-451. doi: https://doi.org/10.15517/REVEDU.V43I2.30068

Rodríguez-Macayo, E., Vidal-Espinoza, R., y Cossio-Bolaños, M. (2019). Desarrollo de las habilidades sociales de estudiantes que ingresan a la universidad. Papeles de trabajo - Centro de Estudios Interdisciplinarios en Etnolingüistica y Antropología Socio-Cultural, (37), 112-128. Recuperado de https://bit.ly/3ein5vH

Pérez, Á. (2009). Las funciones sociales de la escuela: de la reproducción a la reconstrucción critica del conocimiento y la experiencia. Buenos Aires: LPP, Laboratorio de Políticas Públicas. Recuperado de http://biblioteca.clacso.edu.a r/Argentina/lpp/20100324022908/9.pdf

Santamaría-García, H., Baez, S., Gómez, C., Rodríguez-Villagra, O., Huepe, D., y Portela, M. et al. (2020). The role of social cognition skills and social determinants of health in predicting symptoms of mental illness. Translational Psychiatry, 10(1). doi: https://doi.org/10.1038/s41398-020-0852-4

Scott, C. L. (2015). El futuro del aprendizaje 2 ¿Qué tipo de aprendizaje se necesita en el siglo XXI? Investigación y Prospectiva en Educación, 14, 1-19. Recuperado de https://unesdoc.unesco.org/ark:/48223/pf0000242996_sp a/PDF/242996spa.pdf.multi

Segura, M. (2002). Ser persona y relacionarse Habilidades cognitivas y sociales, y crecimiento moral. Madrid, España: Narcea Ediciones.

Torres, D., Fonseca, W., y Pineda, B. (2017). Las vivencias como estrategia de fortalecimiento del pensamiento crítico en educación rural. Praxis \& Saber, 8(17), 201-224. doi: https://dx.doi.org/10.19053/22160159.v8.n17.2018 .7207

Valenzuela-Santoyo, A. y Portillo-Peñuelas, S. (2018). La inteligencia emocional en educación primaria y su relación con el rendimiento académico. Revista Electrónica Educare, 22(3), 228-242. doi: https://dx.doi.org/10.15359/ ree.22-3.11

Vasilachis, I. (2006). Estrategias de Investigación Cualitativa. Barcelona: Gedisa.

Woolfolk, A. (2010). Psicología Educativa. México: Pearson Educación.

\section{INFORMACIÓN ADICIONAL}

Cómo citar: Araneda Machmar, J., Montre Águila, V. (2021). Habilidades sociales en los planes de formación ciudadana de escuelas de las provincias de Concepción y Arauco, región del Biobío-Chile. Revista Educación, 45(2). Recuperado de http://doi.org/10.15517/revedu.v45i1.43561 